\title{
PHOTOEMISSION AND INVERSE PHOTOEMISSION STUDIES OF $\mathrm{SiO}_{2}$
}

\author{
E. SobczaK, R. NiETubyć \\ Institute of Physics, Polish Academy of Sciences \\ Al. Lotników 32/46, 02-668 Warszawa, Poland \\ AND J.W. SOBCZAK \\ Institute of Physical Chemistry, Polish Academy of Sciences \\ Kasprzaka 44/52, 01-224 Warszawa, Poland
}

\begin{abstract}
Occupied and unoccupied electron states of amorphous silicon dioxide film supported on $\mathrm{Si}$ crystal are studied by using X-ray photoemission and, for the first time, X-ray inverse photoemission (X-ray bremsstrahlung isochromat method): A special care was undertaken to minimize decomposition of silicon oxide during X-ray bremsstrahlung measurements. The experimental spectra are compared with theoretical band structure calculations for amorphous $\mathrm{SiO}_{2}$ from the literature and good overall agreement is found.
\end{abstract}

PACS numbers: $71.25 . \mathrm{Tn}, 79.20 . \mathrm{Kz}, 79.60 . \mathrm{Ht}$

\section{Introduction}

Amorphous $\mathrm{SiO}_{2}$ (referred hereafter as a-SiO${ }_{2}$ ), being a wide-gap insulator, is of considerable technological and theoretical interest, therefore a thorough investigation of the electronic states in a- $\mathrm{SiO}_{2}$ is desirable. Vacuum ultraviolet (VUV) and X-ray spectroscopies are very suitable techniques for studies of electronic structure of materials. The occupied part of electron states can be probed by VUV and X-ray photoemission methods giving more or less a total density of states (DOS) in material, whereas X-ray emission band spectra probe a partial DOS and local electronic structure e.g. Si $K_{\beta}$ emission spectrum gives electronic structure of $p$-type valence electrons around $\mathrm{Si}$ ion.

Numerous experimental works on VUV and X-ray photoemission [e.g. 1-3] and X-ray emission spectra [e.g. 4-6] have contributed to a proper understanding of the valence-band structure of $\mathrm{SiO}_{2}$, although much less is known about unoccupied electron bands.

There are a few reports of unoccupied electron states in $\mathrm{SiO}_{2}$ by measuring X-ray absorption spectra at the $\mathrm{Si} K$ edge [7-9], Si $L_{2.3}$ edge [10-12] and $\mathrm{O} K$ 
edge $[10,13,14]$. In general, a synchrotron radiation source is recommended for X-ray absorption studies.

Unoccupied electron states can be also studied by VUV and X-ray bremsstrahlung isochromat method (named inverse photoemission) giving the total density of states weighted by transition probability [15-17]. Surprisingly little was done in this field for $\mathrm{SiO}_{2}$. To our knowledge, only two articles dealing with inverse photoemission studies of $\mathrm{SiO}_{2}$ were published up to now $[18,19]$. Both studies were performed in VUV photon range and for very thin oxide layers on an Si surface. This is partically due to general limitation of the methods applying electron bombardment, which cannot be used for bulk insulators.

In the present paper the electronic structure of amorphous silicon dioxide layer on single crystalline Si was studied by using X-ray photoemission and, for the first time, X-ray inverse photoemission method.

\section{Experimental}

The a-SiO $\mathrm{S}_{2}$ sample was prepared in the Institute of Electronic Materials Technology in Warsaw. The $\mathrm{SiO}_{2}$ film was deposited on a $\mathrm{Si}(111)$ substrate by rf sputtering. The film thickness was about $3000 \AA$. The film obtained was not stoichiometric; the composition $\mathrm{SiO}_{1.85}$ of the layer was established by using an optical reflectivity method. For such a concentration the lattice is essentially that of a- $\mathrm{SiO}_{2}$ with $\mathrm{Si}$ as an impurity [3].

The film thickness of $3000 \AA$ was chosen as being relatively high compared with the mean free path $50 \AA$ for $5400 \mathrm{eV}$ electrons and, on the other hand, relatively low in comparison with the electron penetration depth $0.5 \mu \mathrm{m}$ for $5400 \mathrm{eV}$ electrons in $\mathrm{SiO}_{2}$.

The X-ray inverse photoemission studies were performed by using an isochromat method. In this method an X-ray monochromator was set to pass a narrow band of radiation at a fixed frequency. The intensity of bremsstrahlung radiation was recorded as a function of voltage on the X-ray tube. A Johann monochromator. with $\mathrm{Si}(220)$ bent crystal was set at the photon energy $5415 \mathrm{eV}$. The total spectrometer resolution was $1.8 \mathrm{eV}$. The vacuum in the X-ray tube was of the order of $10^{-6}$ torr. The BIS spectra of a-SiO ${ }_{2}$ layer, as well as for Si crystal and bulk Pd, were measured with $0.5 \mathrm{eV} / 1 \mathrm{~min}$ or $0.5 \mathrm{eV} / 2$ min steps. BIS measurements were performed for a couple of a-SiO ${ }_{2}$ samples to optimize experimental conditions. Several isochromats of each sample were summarized.

\section{Results and discussion}

In Fig. 1 the measured isochromats of a-SiO $\mathrm{S}_{2}$ film and $\mathrm{Si}$ crystal are shown. The BIS intensity is very low, namely 2200 counts $/ 26 \mathrm{~min}$ and 600 counts $/ 8 \mathrm{~min}$ at peak maximum for a- $\mathrm{SiO}_{2}$ and $\mathrm{Si}$ isochromats, respectively. Both isochromats are very different in shape. A strong and broad peak of unoccupied electron states at about $10 \mathrm{eV}$ above the threshold is seen in $\mathrm{a}-\mathrm{SiO}_{2}$ isochromat. The zero energy in the a- $\mathrm{SiO}_{2}$ isochromat was fixed at the Fermi level, which was determined from an isochromat of gold evaporated as a $120 \AA$ layer onto the surface of silicon dioxide. 


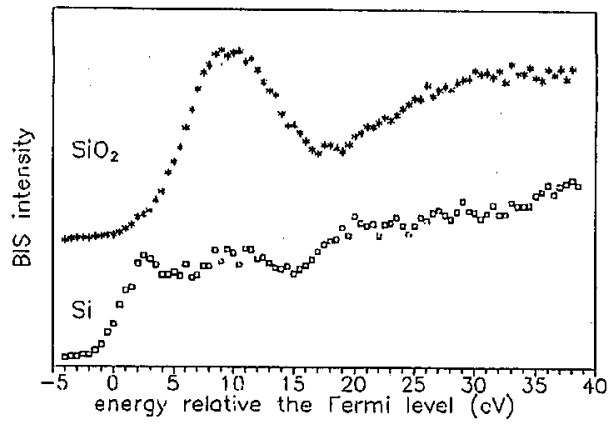

Fig. 1. X-ray bremsstrahlung isochromats of $\mathrm{a}_{-} \mathrm{SiO}_{2}$ and Si-crystal samples at the photon energy $54.15 \mathrm{eV}$.

Within the accuracy of $0.2 \mathrm{eV}$, the energy of Fermi level $\left(E_{\mathrm{F}}\right)$ of silicon dioxide was found to be equal to $E_{\mathrm{F}}$ of the massive $\mathrm{Pd}$. Therefore we concluded that a charge shift of BIS spectrum for our a- $\mathrm{SiO}_{2}$ sample was negligibly small.

A big problem was a decomposition of silicon dioxide during electron bombardment. Without any special thermal contact between the silicon substrate and the water-cooled sample holder, our oxide layer disappeared in twenty hours of electron bombardment at a typical bias of the X-ray tube equal to $5.4 \mathrm{kV}$ and $18 \mathrm{~mA}$ of a beam current. A stripping of an $\mathrm{SiO}_{2}$ film from the Si substrate during electron bombardment was clearly seen in the shape of BIS spectra, which changed continously from a typical $\mathrm{SiO}_{2}$ shape to a form characteristic of Si. We found that a decomposition of $\mathrm{a}-\mathrm{SiO}_{2}$ can be effectively reduced by a good thermal contact between the sample and holder, as well as by decreasing the current of the X-ray tube down to $11 \mathrm{~mA}$. At such conditions the BIS spectrum of a-SiO $\mathrm{S}_{2}$ remained almost unchanged through 140 hours of electron bombardment.

In order to estimate a decomposition rate of $\mathrm{SiO}_{2}$ during BIS rneasurements, $\mathrm{X}$-ray photoelectron spectra of $\mathrm{O} 1 s$ and $\mathrm{Si} 2 p_{3 / 2}$ core levels were studied for the $\mathrm{SiO}_{2}$ layer before and after BIS measurements. X-ray photoelectron measurements were made in a VG ESCALAB 210 spectrometer. We used the $\mathrm{Mg} K_{\alpha}$ excitation $(h \nu=1253.6 \mathrm{eV})$ from an $\mathrm{X}$-ray tube $(15 \mathrm{kV}, 20 \mathrm{~mA})$. The surface was cleaned by argon ion bombardment of $2.5 \mathrm{kV}$ and $5 \mu \mathrm{A}$ during $5 \mathrm{~min}$. We assumed that such sputtering does not introduce any decomposition of the oxide. The $\mathrm{C} 1 \mathrm{~s}, \mathrm{O} 1 \mathrm{~s}$ and Si $2 p$ core levels and valence band photoelectron spectra were recorded before and after ion sputtering.

The XPS O 1 s and $\mathrm{Si} 2 p_{3 / 2}$ core level spectra together with fitting curves are shown in Figs. 2 and 3 . We found that all XPS spectra are shifted to higher binding energies by about $2 \mathrm{eV}$ due to charge effect. The true binding energies were determined, with respect to the Fermi level, assurning the $\mathrm{O} 1 \mathrm{~s}$ binding energy in $\mathrm{SiO}_{2}$ to be equal to $533.3 \mathrm{eV}$ [3]. The results are presented in Table. We estimated the overall accuracy of the XPS measurement to be $0.2 \mathrm{eV}$.

The Si $2 p_{3 / 2}$ core-level spectrum of the investigated a- $\mathrm{SiO}_{2}$ layer (Fig. 3a) was fitted to a single Gaussian-like peak with its FWHM being only $0.1 \mathrm{eV}$ higher than the width of the $\mathrm{O} 1 \mathrm{~s}$ peak. Therefore, we can conclude that a lattice of a- $\mathrm{SiO}_{2}$ 


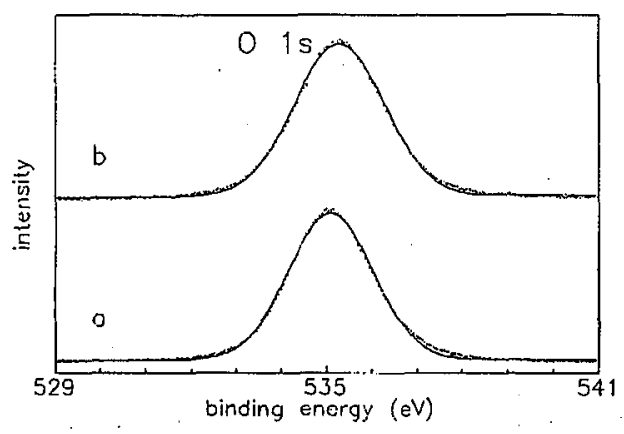

Fig. 2. $\mathrm{O} 1 s$ photoelectron spectra for a-SiO 2 layer: (a) before BIS measurements, (b) after BIS measurements. Experimental data are shown by points and fitting Gaussians are drawn by solid lines. A charge shift of about $2 \mathrm{eV}$ to higher binding energy is visible.

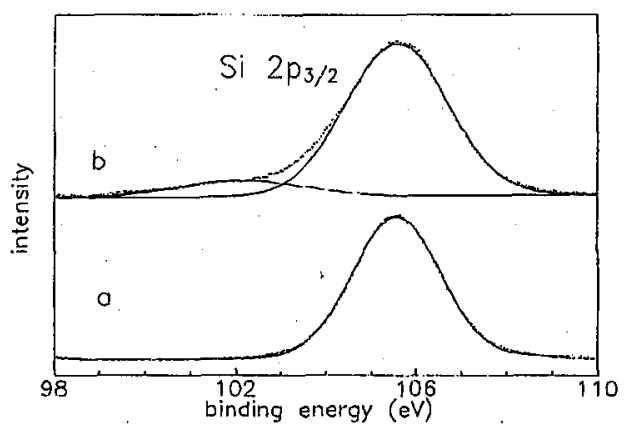

Fig. 3. Si $2 p_{3 / 2}$ plotoelectron spectra for a-SiO 2 layer: (a) before BIS measurements, (b) after BIS measurements. Experimental data are shown by points and fitting Gaussians are drawn by solid lines. A charge shift of about $2 \mathrm{eV}$ to higher binding energy is visible.

TABLE

XPS binding energies and FWHM's of core levels (in eV) for $\mathrm{a}-\mathrm{SiO}_{2}$ before and after long-time BIS measurements.

\begin{tabular}{c|c|c|c|c|c|c}
\hline \hline & \multicolumn{2}{|c|}{ O 1s } & \multicolumn{4}{c}{ Si $2 p_{3 / 2}$} \\
\cline { 2 - 7 } & $E_{\mathrm{B}}$ & FWHM & $E_{\mathrm{B}}$ & FWHM & $E_{\mathrm{B}}$ & FWHM \\
\hline Before BIS & 533.3 & 2.2 & 103.8 & 2.3 & - & - \\
After BIS & 533.3 & 2.3 & 103.6 & 2.7 & 100.1 & 3.2
\end{tabular}

studied consists of tetrahedral bonding $\mathrm{SiO}_{4}$ units with small amount of $\mathrm{Si}_{-} \mathrm{SiO}_{3}$ tetrahedral units of a suboxide. Another Si $2 p_{3 / 2}$ spectrum was observed after long-time electron bombardment (Fig. 3b), where additional small peak (about $10 \%$ of the total integral intensity) appears at a binding energy shifted $3.5 \mathrm{eV}$ 
downward the main peak (compare with Fig. 3a). This broad peak can be atrributed to $\mathrm{Si}_{-} \mathrm{Si}_{4}$ and $\mathrm{Si}-\mathrm{Si}_{3} \mathrm{O}$ tetrahedra [3]. We also obtained that the main peak Si $2 p_{3 / 2}$ became wider by $0.4 \mathrm{eV}$ and shifted to the lower binding energy by $0.2 \mathrm{eV}$ (see Table) after electron bombardment. It can be ascribed to the increasing concentration of $\mathrm{Si}-\mathrm{SiO}_{3}$ tetrahedra in comparison with that of $\mathrm{SiO}_{4}$ tetrahedra.

The XPS valence band spectrum of $\mathrm{a}_{-} \mathrm{SiO}_{2}$ film was measured in a binding energy range from 40 to $-5 \mathrm{eV}$, with $0.05 \mathrm{eV} / 0.2 \mathrm{~s}$ steps, through 150 scans. The maximal intensity in this region was 120000 counts/30 s. The XPS valence band spectrum was corrected for $\mathrm{Mg} K_{\alpha_{3,4}}$ satellites and charge shift. The corrected photoemission valence band spectrum and inverse photoemission spectrum were put together in a common energy scale in Fig. 4. A photoelectron spectrum of a- $\mathrm{SiO}_{2}$

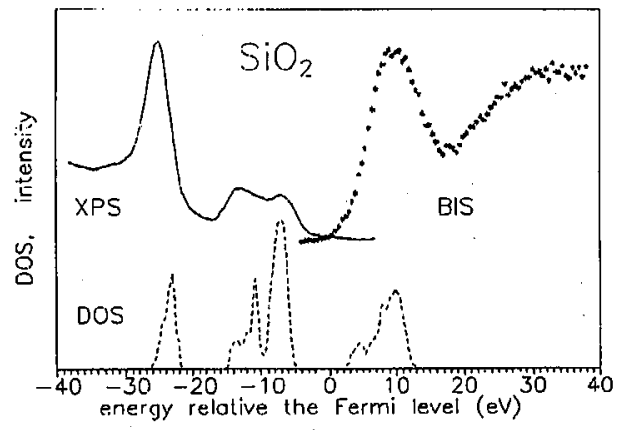

Fig. 4. Valence band photoemission and X-ray inverse photoemission spectra of a- $\mathrm{SiO}_{2}$ on a common energy scale as discussed in the text. Below the theoretical DOS of a- $\mathrm{SiO}_{2}$ $[20]$ is shown.

displays three clearly discernible features. The peak at binding energy of about $-25 \mathrm{eV}$ refers to the $\mathrm{O} 2 s$ orbital. The $\mathrm{O} 2 p-\mathrm{Si} 3 p, 3 s$ bonding orbital appears at binding energy $-13 \mathrm{eV}$, and the $\mathrm{O} 2 p$ nonbonding orbital is found at binding energy $-5 \mathrm{eV}$ at the valence band top. The inverse photoemission spectrum displays two broad maxima at 10 and $30 \mathrm{eV}$. In molecular approach the first one is an O $2 p-\mathrm{Si} 3 s, 3 p$ antibonding orbital, while the second one is discussed below as $\sigma$-resonance. In Fig. 4the theoretical total density of electron states for a- $\mathrm{SiO}_{2}$ calculated by Ching [20] is also shown. An energy gap of about $9 \mathrm{eV}$ between the photoemission and inverse photoemission peaks is visible. The distance $17 \mathrm{eV}$ between maxima of those peaks for a- $\mathrm{SiO}_{2}$ layer studied here is in good agreement with theoretical model for amorphous $\mathrm{SiO}_{2}[20]$.

The XPS emission from $\mathrm{O} 2 s$ level is strong in comparison with those peaks from higher-lying $\mathrm{O} 2 p$ orbitals. Also the XPS intensity of $\mathrm{O} 2 p$ nonbonding orbital seems relatively smaller than it could result from the calculations of DOS (Fig. 4). These amplitude modifications are due to the energy dependent transition probabilities [21], which weight the components of the photoemission spectrum. Thus, the observed spectrum does not reproduce the true density of states, but it only shows a weighted density of states.

The energy dependence of BIS transition probability closely resembles that of 
photoemission: the $l$-partial transition probability decreases with increasing photon energy and the rate of decrease becomes larger with the $l$ number [22]. We estimated that relative probabilities of X-ray bremsstrahlung transitions to final electron states $\mathrm{O} 3 s, \mathrm{O} 3 p, \mathrm{Si} 3 s, \mathrm{Si} 3 p$ and $\mathrm{Si} 3 d$ for photon energy $5415 \mathrm{eV}$ are equal to $1 / 6,1 / 100,1,1 / 9$ and $1 / 1000$, respectively. For the estimation we used data calculated in Coulombic approxirnation by Hahn et al. [23] and a scaling procedure proposed by these authors. Therefore, the probability of BIS transitions to $\mathrm{Si} s$-like states located at the bottom of conduction band of $\mathrm{SiO}_{2}$ is higher than this of transitions to Si $p$-like states placed few $\mathrm{eV}$ above the bottom. On the other hand, the $\mathrm{O} s$-like and $\mathrm{O} p$-like contributions to BIS intensity are small in the range studied due to low local density of unoccupied electron states around oxygen ions in comparison with silicon ions, according to theoretical models, which predict that the occupied electron states are localized at oxygen ions, while the unoccupied electron states at the silicon ions $[20,24]$. Therefore, in the first approximation the BIS spectrum of $\mathrm{SiO}_{2}$ should reflect the total density of unoccupied states with an enhancement of Si s-type states contributions.

A second BIS maximum appears at the energy of $30 \mathrm{eV}$ above the Fermi level, i.e. about $20 \mathrm{eV}$ above the first BIS maximum. This energy distance agrees well with the energy of plasmon loss in $\mathrm{SiO}_{2}\left(E_{\mathrm{p}}=21 \mathrm{eV}\right)$ evaluated from the XPS spectra. We suggest that the second maximum originates rather from the electronic structure than from a plasmon loss, because a similar peak was observed in the absorption spectra at about $20 \mathrm{eV}$ above the "white" line $[9,10,12,14]$ and was interpreted as a $\sigma$-resonant scattering of photoelectrons by adjacent atoms $[14,25]$.

In conlusion, a complementary study of both occupied and unoccupied electron states in amorphous $\mathrm{SiO}_{2}$ by using $\mathrm{X}$-ray photoemission (XPS) and, for the first time, by $X$-ray inverse photoemission (BIS) has been performed. We have aligned the photoemission valence band spectrum with BIS spectrum in a comrion energy scale. Our experimental results are consistent with the rnodel calculations of density of states for $\mathrm{a}_{-} \mathrm{SiO}_{2}$ by Ching [20]. We have performed an analysis of the Si $2 p$ and $\mathrm{O} 1 \mathrm{~s}$ core-level spectra for samples before and after BIS measurements, which showed a partial decomposition of $\mathrm{SiO}_{2}$ due to a long-time electron bombardment, during BIS experiment.

\section{Acknowledgment}

This work was supportied by the project No. 2P302 25604 of the State Committee for Scientific Research (Republic of Poland).

\section{References}

[1] T.H. DiStefano, D.E. Eastman, Phys. Rev. Lett. 27, 1560 (1971).

[2] G. Hollinger, Appl. Surf. Sci. 8, 318 (1981).

[3] F.G. Bell, L. Ley, Phys. Rev. B 37, 8383 (1988).

[4] G. Klein, H.-U. Chun, Phys. Status Solidi B 49, 167 (1972).

[5] G. Wiech, Solid State Commun. 52, 807 (1984). 
[6] J. Nithianandam, S. Schmatterly, Phys. Rev. B 40, 11786 (1989).

[7] C. Senemaud, M.T. Costa Lima, J.A. Roger, A. Cachard, Chem. Phys. Lett. 26, 431 (1974).

[8] G.N. Greaves, A. Fontaine, P. Lagarde, D. Raoux, S.J. Gurman, Nature 293, 611 (1981).

[9] M.R. Caroll, E. Paris, I. Davoli, in: Proc. 2nd European Conf. on Progress in $X$-ray Synchrotron Radiation Rescarch, Eds. A. Balema, E. Bernieri, S. Mobilio, SIF, Bologna 1990, p. 647.

[10] O.A. Ershov, A.P. Lukirskii, Fiz. Tverd. Tela 8, 2137 (1966).

[11] Y. Iguchi, K.P. Miyake, J. Phys. Soc. Jpn. 33, 273 (1972).

[12] F.C. Brown, R.Z. Bachrach, M. Skibowski, Phys. Rev. B 15, 4781 (1977).

[13] J. Stöhr, L. Johansson, I. Lindan, P. Pianetta, Phys. Rev. B 20, 664 (1979).

[14] G.E. Brown Jr, G.A. Waychunas, J. Stohr, F. Sette, J. Phys. (France) C 8, supl. 12, 685 (1986).

[15] E. Sobczak, J. Auleytner, Phys. Rev. B 37, 6251 (1988).

[16] E. Sobczak, Acta Phys. Pol. A 83, 135 (1993).

[17] J.C. Fuggle, in: Unoccupied Electronic States, Eds. J.C. Fuggle, J.E. Inglesfield, Springer-Verlag, Berlin 1992, p. 307.

[18] F.J. Himpsel, D. Straub, Surf. Sci. 168, 764 (1986).

[19] M. Azizan, R. Baptist, A. Brenac, G. Chauvet, T.A. Nguyen Tan, J. Phys. (France) 48, 81 (1987).

[20] W.Y. Ching, Phys. Rev. B 26, 6622 (1982).

[21] J.J. Yeh, I. Lindau, At. Data Nucl. Data Tables 32, 1 (1985).

[22] A. Simunek, J. Vackar, E. Sobczak, Phys. Rev. B 38, 8515 (1988).

[23] Y. Hahn, D.W. Rule, J. Phys. B, At. Mol. Phys. 10, 2689 (1977).

[24] R.P. Gupta, Phys. Rev. B 32, 8278 (1985).

[25] A.P. Hitchock, F. Sette, J. Stöhr, in: EXAFS and Near Edge Structure III, Eds. K.O. Hodgson, B. Hedman, J.E. Penuer-Hahn, Springer Verlag, Berlin 1984, p. 43. 